\title{
LH and FSH secretory responses to GnRH in normal individuals: a non-parametric deconvolution approach
}

\author{
Giuseppe De Nicolao $^{1}$, Diego Liberati ${ }^{2}$, Johannes D Veldhuis ${ }^{3}$ and Alessandro Sartorio ${ }^{4,5}$ \\ ${ }^{1}$ Dipartimento di Informatica e Sistemistica, Università di Pavia, Via Ferrata 1, 27100 Pavia, Italy, ${ }^{2}$ Consiglio Nazionale delle Ricerche, Istituto \\ Circuiti Elettronici,Via De Marini 6, 16149 Genova, Italy, ${ }^{3}$ Division of Endocrinology and Metabolism, University of Virginia Health Sciences Center, \\ NSF Center for Biological Timing, Charlottesville, Virginia 22908, USA, ${ }^{4}$ Divisione Malattie Metaboliche III, Istituto Auxologico Italiano, IRCCS, \\ Piancavallo (VB), Italy and ${ }^{5}$ Laboratorio Sperimentale di Ricerche Endocrinologiche, Istituto Auxologico Italiano, IRCCS, Milano, Italy \\ (Correspondence should be addressed to G De Nicolao; Email: denicolao@conpro.unipv.it)
}

\begin{abstract}
Objective: To reconstruct the instantaneous secretion rate (ISR) of LH and FSH after GnRH administration in normal volunteers using non-parametric deconvolution, and to derive a direct integration formula to evaluate the amount of LH and FSH secreted during the first 60 min after the stimulus.

Design and methods: First, the deconvolution method was validated in vivo by reconstructing doses ranging from $7.5 \mathrm{IU}$ to $75 \mathrm{IU}$ injected in three healthy adult volunteers whose endogenous LH had previously been downregulated by pretreating them, 3-4 weeks earlier, with $3.75 \mathrm{mg}$ GnRH agonist i.m. Then, 40 healthy adult male volunteers were tested with a single $100 \mu \mathrm{g} \mathrm{GnRH}$ bolus, administered at $0 \mathrm{~min}$. LH and FSH concentrations were determined at $-30,0,15,30,45,60,90$, and $120 \mathrm{~min}$.

Results and conclusions: The validation study, conducted over a 10-fold range of doses, demonstrated that non-parametric deconvolution provided a reasonably accurate estimate of the amount of hormone entering the circulation. Applying deconvolution to the LH and FSH responses to GnRH, the ISRs of both hormones were shown to have a similar pattern, with a clearly delimited pulse after the GnRH bolus. In conjunction with earlier analyses of estimates of GHRH-stimulated GH secretion, we conclude that secretagogues evoke discrete LH, FSH, and GH secretory bursts of about 60 min total duration, despite markedly unequal (glyco-)protein hormone half-lives (18-500 min). With respect to the assessment of total hormone release during the first $60 \mathrm{~min}$ after the stimulus, the integration formula provided a reliable approximation of the result obtained by deconvolution, and had a negligible dependence on the samples at times 90 and $120 \mathrm{~min}$.
\end{abstract}

European Journal of Endocinology $141246-256$

\section{Introduction}

The responsiveness of the hypophysis to a gonadotropinreleasing hormone $(\mathrm{GnRH})$ stimulus is usually assessed by collecting samples for serum luteinizing hormone (LH) and follicle-stimulating hormone (FSH) determinations over the ensuing hours. The analysis of such response-to-stimuli (RTS) data poses some unsolved problems, in that the serum concentration profile reflects only indirectly the secretory activity elicited by the stimulus. In fact, because of clearance effects, at each instant in time, the serum concentration depends on all the glandular secretory activity that occurred beforehand. As a consequence, a greater peak value of the concentration does not necessarily correspond to a greater response in terms of released hormone. Also the incremental area under the curve (AUC) is not a reliable index of responsiveness, because the usual sampling procedures (lasting about $2 \mathrm{~h}$ ) do not capture the full descending limb of the $\mathrm{LH}$ and FSH responses. This implies that the incremental AUC is not proportional to the amount of released hormone. Moreover, the final part of the concentration profile may be affected also by spontaneous secretion pulses, which could further bias the AUC. In addition, secretory responses of two hormones with dissimilar half-lives (e.g. LH and FSH) cannot be compared directly by AUC after a single relevant stimulus $(\mathrm{GnRH})$, because of the strong bias introduced by the half-life differences.

A possible way to overcome the above problems is to reconstruct the instantaneous secretion rate (ISR) of the hypophysis, using the serum hormone concentration profile and a notion of the hormone kinetics. This is a so-called 'inverse problem', in which, given the observed effects (the serum LH or FSH concentrations), a mathematical algorithm is used to back-calculate the cause (the LH or FSH glandular ISR). As the direct formula to compute the serum concentration from the ISR is a convolution integral, the process of recovering the ISR from the observations is called deconvolution. In 
the past decade, deconvolution analysis of hormone data has been the subject of several studies (1-5). However, some of the parametric and discrete deconvolution techniques proposed in the literature were developed for the analysis of spontaneous secretion data and are not optimal to deal with the analysis of RTS data, for example those obtained in GnRH tests. In fact, because the shape of the ISR profile is unknown, it may not be desirable to adopt parametric methods, which require explicit assumptions about the mathematical waveform of the secretory profile. Likewise, approximating the ISR as a constant in between the sampling instants, as is done in discrete deconvolution, is unsatisfactory when the sampling intervals are infrequent (6). Thus several waveform-independent approaches have been proposed (5).

More recently, the introduction of non-parametric deconvolution algorithms (5-8) has made it possible to obtain smooth non-negative estimates of the ISR in RTS experiments, without introducing assumptions as to shape. So far, the application of these techniques to RTS data has been limited to the analysis of growth hormone (GH) responses to single and repeated GH-releasing stimuli (8). The purpose of this study was to provide an 'in vivo' validation of the non-parametric deconvolution method and then investigate its applicability to the assessment of LH and FSH responses after GnRH stimuli. A major difference with respect to $\mathrm{GH}$ experiments is the longer half-life of LH and especially FSH (about $10 \mathrm{~min}$ for $\mathrm{GH}, 30 \mathrm{~min}$ for $\mathrm{LH}$ and $200 \mathrm{~min}$ for $\mathrm{FSH}$ ). Therefore, as distinguished from $\mathrm{GH}$ data, for which the shape of the ISR closely resembles the serum GH concentration profile, the LH and FSH concentration responses are expected to be much more prolonged than the underlying ISR pulses.

\section{Methods}

\section{Participants}

Forty healthy adult male volunteers (age range: 2237 years) were admitted to the study; all were of normal height and weight. The procedure was approved by the Ethics Committee of the Italian Institute for Auxology, Milan; informed consent was obtained in all cases.

The volunteers were tested with a single $100 \mu \mathrm{g}$ GnRH bolus (LHRH; Ferring, Kiel, Germany), administered at $0 \mathrm{~min}$; $\mathrm{LH}$ and FSH concentrations were determined at $-30,0,15,30,45,60,90$, and $120 \mathrm{~min}$. All tests started between $0800 \mathrm{~h}$ and $0830 \mathrm{~h}$, after the individual had fasted overnight.

Serum LH and FSH concentrations were measured using commercial RIA kits (Ciba Corning Diagnostics, Walpole, USA); the sensitivity of the method was 0.07 and $0.3 \mathrm{IU} / \mathrm{l}$ for $\mathrm{LH}$ and FSH respectively. The total coefficients of variation (CV) were 6.3, 6.2, 6.3 and $5.2 \%$ at LH concentrations of $5.3,7.2,14.8$ and
36.5 IU/l; 4.6, 4.5 and $4.7 \%$ at FSH levels of 4.3, 20.9 and $45.1 \mathrm{IU} / \mathrm{l}$. All samples were analysed at the same time.

In order to perform an 'in vivo' validation of the deconvolution method used to analyse the previous data, three healthy male volunteers (weights: $86 \mathrm{~kg}$, $68 \mathrm{~kg}, 86 \mathrm{~kg}$ ), recruited at the Division of Endocrinology and Metabolism of the University of Virginia, were injected with leuprolide acetate $3.75 \mathrm{mg}$ i.m., and 2128 days later underwent blood sampling at 10-min intervals beginning at $0800 \mathrm{~h}$. After the first blood sample was obtained, human recombinant LH (Serono Laboratories, Norwall, MA, USA) was infused over 1 or $8 \mathrm{~min}$ i.v. every $2 \mathrm{~h}$ in the dose concerned, in order to emulate pulsatile hormone production. Five experiments were carried out on separate mornings with the same procedure but different doses, namely 7.5, 15, 30, 50 and $75 \mathrm{IU}$. The doses were given as 1-min infusions to the first volunteer in the first three experiments, each of which lasted $8 \mathrm{~h}$, corresponding to four artificial pulses. Conversely, 8-min infusions were adopted in the other two experiments, each of which lasted $16 \mathrm{~h}$, corresponding to eight artificial pulses. Serum was later assayed for LH content by twosite immunoradiometric assay, as described previously (9).

\section{Deconvolution analysis}

The plasma LH concentration (c) (IU/l) has been described using the following convolution integral (3):

$$
c(t)=\int_{-\infty}^{t} g(t-\tau) s(\tau) \mathrm{d} \tau
$$

where $g(t)$ denotes the LH elimination function and $s(\tau)$ (IU/(l min)) is the LH ISR, normalized by the distribution volume. The function $g(t)$ has been modelled as the sum of two exponentials (10):

$$
g(t)=a \exp (-\alpha t)+(1-a) \exp (-\beta t), t \geq 0
$$

where $a=0.62 \pm 0.1722, \alpha=0.0387 \pm 0.012 \mathrm{~min}^{-1}$ and $\beta=0.00769 \pm 0.0015 \mathrm{~min}^{-1}$ (mean \pm S.D.). These are population parameters estimated in a previous study (10) by fitting the two-exponential model to the plasma disappearance profiles observed after bolus i.v. injection of $35 \mu \mathrm{g} \mathrm{LH}$. As for $\mathrm{FSH}$, the same biexponential model has been adopted, with $a=0.52$, $\alpha=0.0068 \mathrm{~min}^{-1}$ and $\beta=0.0014 \mathrm{~min}^{-1}$. Again, these are population parameters estimated in a previous study (11) by fitting the two-exponential model to the plasma disappearance profiles observed after bolus i.v. injection of FSH (250 IU). With regard to the applicability of these parameters to the present data, note that the disappearance profiles in the earlier studies $(10,11)$ have the same order of magnitude as the responses analysed in the present study (compare, for instance 
Fig. 1 in (10) and Fig. 3 in (11) with Fig. 2 later in the present paper).

In the analysis, only measured (noisy) values of plasma concentration $y_{k}(\mathrm{IU} / \mathrm{l}), k=1, \ldots, n$, are available:

$$
y_{k}=c\left(t_{k}\right)+v_{k}
$$

where $v_{k}$ denotes additive (uncorrelated) zero-mean noise with concentration-dependent variance.

For LH data, the error model

$$
\operatorname{Var}\left[v_{k}\right]=a_{1} c\left(t_{k}\right)^{a_{2}}
$$

where $a_{1}=0.0057$ and $a_{2}=1.8140$, was derived via least squares fitting using the $\mathrm{CV}$ values at 5.3, 7.2, 14.8 and $36.5 \mathrm{IU} / \mathrm{l}$ reported in the previous section.

For FSH, a constant CV error model was used:

$$
\operatorname{Var}\left[v_{k}\right]=\left(4.6 c\left(t_{k}\right)\right)^{2}
$$

The mathematical and statistical foundations of the non-parametric deconvolution method used in this paper have been thoroughly discussed (7) and its use for the estimation of the GH secretory rate from RTS data has been illustrated previously (8). For the sake of consistency, a concise description of the basic principles is now reported.

Non-parametric deconvolution allows us to reconstruct the continuous-time function $s(\tau)$ from discrete and noisy samples of hormone concentration, without introducing arbitrary assumptions ('prejudices') on the shape of the ISR. According to the Phillips-Tikhonov regularization method $(12,13)$, we introduce the score function:

$$
J(s)=\sum_{j=1}^{n} \frac{\left(y_{j}-c\left(t_{j}\right)\right)^{2}}{\operatorname{Var}\left[v_{j}\right]}+\gamma \int_{-\infty}^{t_{n}}\left(\frac{\mathrm{d} s(t)}{\mathrm{d} t}\right)^{2} \mathrm{~d} t
$$

This function is the sum of a weighted least-squares fit plus a 'smoothness' penalty given by the integral of the squared derivative of $s(t)$, which is large if $s(t)$ is 'irregular' (i.e. wiggly). The balance between the two terms is controlled by the positive real number $\gamma$, the socalled regularization parameter, the optimal value of which is found via the maximum likelihood principle. The idea is that a 'good' estimate $s(t)$ should yield a small value of the score function $J(s)$. In particular, the best ISR profile $s(t)$ is the one that yields the minimum possible value for $J(s)$ (it can be shown that such a profile is unique). Note that it would not be possible to consider least squares minimization alone, because there are infinitely many ISR profiles $s(t)$ that annihilate the sum of squared residuals. Once $\gamma$ has been estimated, a non-negative solution $s(\mathrm{t})$ is obtained by minimizing $J(s)$ using a constrained conjugate gradient method that forces the solution $s(t)$ to be non-negative. To allow for the sudden change of slope that occurs in the ISR in correspondence with the GnRH stimulus, a slightly modified version of the regularization method (initially proposed and validated on $\mathrm{GH}$ data (8)) has been adopted.
Although an explicit formula for computing the confidence interval is available, it relies on the assumption that the model of the hormone kinetics is perfectly known, which does not apply when, as in the present study, population kinetic estimates are used. Then, more realistic confidence intervals, allowing for both measurement errors and model uncertainty, are obtained by resorting to a Monte Carlo procedure (7). The data and clearance parameters are randomly perturbed in order to emulate measurement and inter-volunteer variability. By solving several perturbed deconvolution problems, one obtains a set of perturbed solutions, the distribution of which reflects the variability of the estimates. In the present study, 200 Monte Carlo runs were performed for each volunteer, to compute asymmetric confidence intervals. As the ISR is a continuous function of time, the use of upper and lower limits representing pointwise error bars is not completely satisfactory. To visualize better how uncertainty is distributed over the whole ISR profile, a number of estimates (say 10) are extracted from the 200 estimated ISRs and are jointly plotted in the same graph. When the Monte Carlo runs were performed for FSH, the parameters $a, \alpha$ and $\beta$ each had the same $\mathrm{CV}$ as the corresponding parameter for $\mathrm{LH}$, namely $28 \%, 31.01 \%$; and $19.51 \%$.

\section{A direct integration formula for secretion assessment}

If one is interested in assessing the responsiveness of $\mathrm{LH}$ (or FSH) to a GnRH stimulus at time $0 \mathrm{~min}$, an appropriate index is given by the amount $\mathrm{H}$ (IU/l) of hormone secreted (per volume) over the time interval $[0, L]$ (e.g. $L=60 \mathrm{~min})$. Such an amount is simply the AUC of the ISR, which can be evaluated as:

$$
H=\int_{0}^{L} \hat{s}(t) d t
$$

where $\hat{s}(t)$ is the ISR estimated by deconvolution. Now, we introduce an approximation by zeroing the regularization parameter and neglecting non-negativity and discontinuities. Then, an explicit formula is available that gives $\hat{s}(t)$ as a linear function of the concentration data (6). By inserting this formula into equation [3], after some computations (see Appendix), we obtain the following approximate integration formula for calculating $H$, without the need explicitly to perform deconvolution:

$$
H \cong \theta_{1} y_{1}+\theta_{2} y_{2}+\ldots+\theta_{n} y_{n}
$$

where $\theta_{1}, \theta_{2}, \ldots, \theta_{n}$ are suitable weightings that depend on: (i) the clearance parameters of the specific hormone; (ii) the sampling schedule; (iii) the length, $L$, of the time interval. The weightings for $\mathrm{LH}$ and $\mathrm{FSH}$ with $L=60 \mathrm{~min}$ relative to the two sampling schedules $(-30,0,15,30,45,60,90,120 \mathrm{~min}$ and $-30,0$, $15,30,45,60 \mathrm{~min})$ are reported in Table 1. For FSH 
Table 1 Weightings $(\theta)$ for computing the amount of secreted hormone, $H(\mathrm{IU} / \mathrm{I})$, over the time interval $0-60$ min according to the direct integration formula, $H \cong \theta_{1} y_{1}+\theta_{2} y_{2}+\ldots$, where $y_{k}$ denotes the hormone concentration in plasma at time $t_{k}$.

\begin{tabular}{|c|c|c|c|c|c|c|c|c|}
\hline \multirow[b]{2}{*}{ Hormone } & \multicolumn{7}{|c|}{ Time (min) } & \multirow[b]{2}{*}{$\begin{array}{c}120 \\
\theta_{8}\end{array}$} \\
\hline & $\begin{array}{c}-30 \\
\theta_{1}\end{array}$ & $\begin{array}{c}0 \\
\theta_{2}\end{array}$ & $\begin{array}{l}15 \\
\theta_{3}\end{array}$ & $\begin{array}{l}30 \\
\theta_{4}\end{array}$ & $\begin{array}{l}45 \\
\theta_{5}\end{array}$ & $\begin{array}{l}60 \\
\theta_{6}\end{array}$ & $\begin{array}{l}90 \\
\theta_{7}\end{array}$ & \\
\hline $\begin{array}{l}\text { LH } \\
\text { LH } \\
\text { FSH } \\
\text { FSH }\end{array}$ & $\begin{array}{l}-0.3510 \\
-0.3509 \\
-0.0741 \\
-0.0741\end{array}$ & $\begin{array}{l}-0.8912 \\
-0.8916 \\
-0.9737 \\
-0.9741\end{array}$ & $\begin{array}{l}0.3799 \\
0.3817 \\
0.1174 \\
0.1192\end{array}$ & $\begin{array}{l}0.2825 \\
0.2756 \\
0.0245 \\
0.0176\end{array}$ & $\begin{array}{l}0.4759 \\
0.5022 \\
0.1459 \\
0.1719\end{array}$ & $\begin{array}{l}1.1046 \\
1.0660 \\
0.9762 \\
0.9425\end{array}$ & $\begin{array}{c}-0.0233 \\
- \\
-0.0169 \\
-\end{array}$ & $\begin{array}{l}0.0047 \\
- \\
0.0036 \\
-\end{array}$ \\
\hline
\end{tabular}

also the case of only two samples at 0 and $60 \mathrm{~min}$ was considered: when $L=60 \mathrm{~min}$, the corresponding weightings are $\theta_{1}=-0.9281$ and $\theta_{2}=1.1402$.

\section{Simulated secretion data}

To validate the deconvolution technique mathematically, two simulated benchmark problems were devised. An artificial ISR signal, which reproduced the (likely) secretory response to a GnRH stimulus at time 0, was defined as:

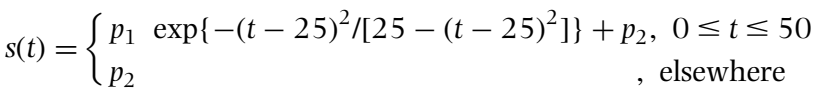

with either $p_{1}=1.5, p_{2}=0.1 \quad(\mathrm{LH})$ or $p_{1}=0.2$, $p_{2}=0.02(\mathrm{FSH})$. The parameters $a, \alpha$ and $\beta$ for LH and FSH were assumed to be as previously reported.

Computing the convolution of the impulse response with the artificial ISR signal, a simulated LH concentration profile and a simulated FSH peak were obtained. Observations of the output $y(t)$ were assumed to be available at times $-30,0,15,30,45,60,120 \mathrm{~min}$. In order to simulate measurement errors, artificial noise, having the same concentration-dependent variance as reported above for the deconvolution analysis, was added to the concentration samples. (The serum LH concentration profiles and its ISR are shown later, in Fig. 2.) To simulate imperfect knowledge of the kinetic model, deconvolution was performed using the nominal parameters:

$$
\begin{gathered}
a=0.558, \alpha=0.03483 \mathrm{~min}^{-1}, \\
\beta=0.008459 \mathrm{~min}^{-1} \quad(\mathrm{LH}) \\
a=0.486, \alpha=0.00477 \mathrm{~min}^{-1}, \\
\beta=0.0001804 \mathrm{~min}^{-}
\end{gathered}
$$

that were different from the ones used to produce the observations.

\section{Results}

\section{In vivo validation of deconvolution}

The data from the five validation experiments underwent non-parametric deconvolution in order to estimate the corresponding ISR. Inspection of both the original data and the estimated ISR profiles revealed that spontaneous secretion, though downregulated, was not completely absent. Then, in order to isolate the injected doses from spontaneously secreted hormone, the ISR was integrated over intervals ranging from $10 \mathrm{~min}$ before the beginning of infusions to $30 \mathrm{~min}$ after. An estimate of the injected dose was then obtained by multiplying the integrated ISR by the plasma volume. The plasma volume was estimated as $7 \%$ body weight. In Fig. 1, the injected doses are plotted against the doses reconstructed by deconvolution (average values). In the ideal case, the points should lie on the diagonal, although, in practice, this can hold only approximately, because of the effect of measurement errors, kinetic parameter uncertainty and uncertainty in the determination of the blood volume. Given all these sources of errors, non-parametric deconvolution reconstructs the true doses very satisfactorily.

\section{Analysis of simulated data}

The LH ISR of the benchmark problem estimated by means of regularized non-parametric deconvolution (with discontinuity of the first derivative at time $0 \mathrm{~min}$ ) is shown in Fig. 2A. The root-mean-square (RMS) error between the true and the estimated ISR was $0.1263 \mathrm{IU} /$ (l min), which is less than $10 \%$ of the peak value. Given that only eight samples are available, the accuracy of the estimate is rather good. By reconvolving the estimated ISR, an estimate of the serum LH concentration profile was obtained (Fig. 2B). Note the different shape of the serum profile and the ISR: although the concentration data capture only the first part of a long and skewed pulse, the deconvolved secretion estimate recovers the original shorter ISR pulse. Figure 2C shows 10 ISR profiles taken from the 200 profiles computed in the Monte Carlo analysis (see Methods). This kind of plot provides a more faithful representation of the actual variability of the estimated ISR compared with pointwise confidence limits.

Deconvolution of the simulated FSH data was also performed. The RMS error between the true and the estimated ISR was $0.0211 \mathrm{IU} /(\mathrm{l} \mathrm{min})$. Again, this is about 


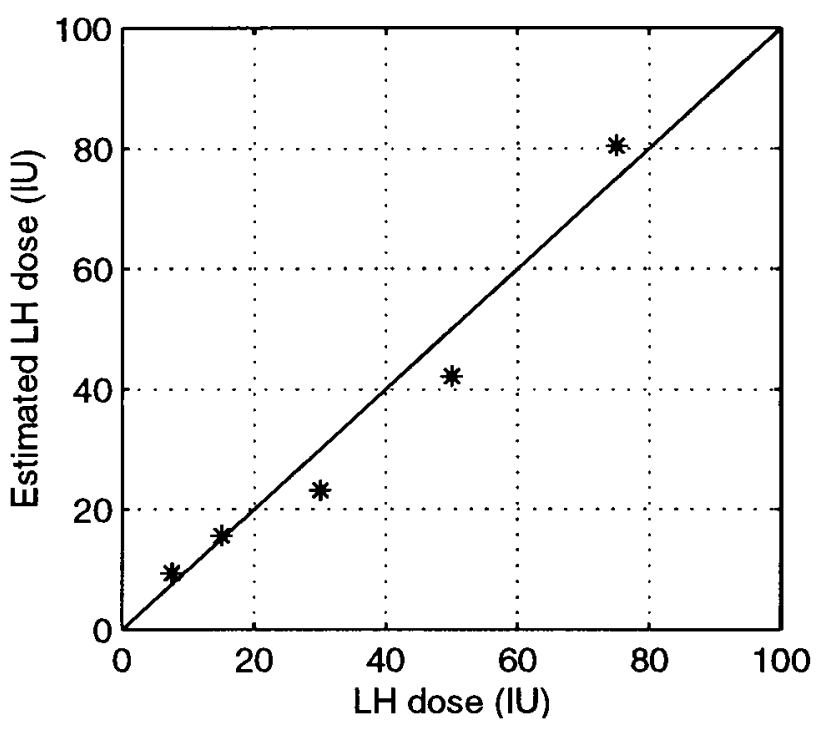

Figure 1 Recombinant human LH infusions over 1 min $(7.5,15$ and $30 \mathrm{IU}$ doses) or over $8 \mathrm{~min}$ (50 and $75 \mathrm{IU}$ doses) in leuprolidedownregulated men (pretreated 3-4 weeks earlier with $3.75 \mathrm{mg}$ $\mathrm{GnRH}$ agonist i.m.). LH was injected at the given doses every $2 \mathrm{~h}$. Data are LH concentrations in blood sampled every $10 \mathrm{~min}$. The figure shows the comparison between the actual doses (horizontal axis) and the doses estimated by applying deconvolution to the blood LH concentration data (vertical axis). In the ideal case, the points should lie on the diagonal.

$10 \%$ of the peak value. The results (not shown) are equivalent to those relative to the simulated $\mathrm{LH}$ data.

\section{Analysis of experimental data}

Non-parametric deconvolution analysis was applied to the experimental $\mathrm{LH}$ and $\mathrm{FSH}$ data $(\mathrm{GnRH}$ bolus at $0 \mathrm{~min}$ ). The results in a representative case (volunteer 3 ) are depicted in Fig. 2 for both LH and FSH. In panels D-E (LH) and G-H (FSH), the estimated ISR and the serum hormone concentrations are plotted, together with their 95\% confidence limits, computed taking into account both measurement error and kinetic model uncertainty. In panel E, (LH) and panel I (FSH), 10 ISR profiles taken from the 200 profiles of the Monte Carlo analysis are presented. The estimated LH and FSH ISRs in volunteers Nos $6,11,13,14,18,19,20,23,26,30,33$ and 34 . are reported for comparison in Figs 3 and 4.

The GnRH injection caused a clear increase in serum $\mathrm{LH}$ and FSH concentrations in all volunteers. The application of deconvolution showed an increase also in the estimated ISR. Because of the clearance effect, $120 \mathrm{~min}$ post-stimulus was an insufficient time-frame in which to observe the complete decay of the serum concentration pulse, and only the first part of the typical peaked profile was visible. However, although the serum hormone concentration had not returned to baseline values at time $120 \mathrm{~min}$, deconvolution analysis showed the complete transient of the ISR response which, after a distinct peak, in most cases decreased towards normal values within about $60 \mathrm{~min}$ of the stimulus. This suggests that it may be possible to assess the LH and FSH response using samples collected for only $60 \mathrm{~min}$ after GnRH. To test this possibility, the samples at time 90 and $120 \mathrm{~min}$ were discarded, and deconvolution performed using only the remaining six samples at times $-30,0,15,30,45,60 \mathrm{~min}$. Then, the cumulated ISRs over 0-60 min were compared in the two cases (using six and eight samples); as depicted in Fig. 5, the difference is negligible. The abscissae of the 40 plotted points in Fig. 5A represent the values of the estimated cumulative secretion of LH over $1 \mathrm{~h}$ (range 15-157 U/l; average $51 \pm 28 \mathrm{U} / \mathrm{l}$ (mean \pm s.D. $)$ ). Conversely, the abscissae of the 40 plotted points in Fig. 5B represent the values of the estimated cumulative secretion of FSH over $1 \mathrm{~h}$ (range $0.6-19.6 \mathrm{U} / \mathrm{l}$; average $7.8 \pm 4.6 \mathrm{U} / \mathrm{l}$ (mean \pm S.D. $)$ ).

The amount of hormone secreted over 0-60 min was also evaluated using the direct integration formula (eq. [4]). The comparison with the values obtained from the deconvolved ISR is summarized in Fig. $5 \mathrm{C}$ and D. The eight-sample integration formula yields very accurate results. Inspection of the weightings used in the integration formula (Table 1) confirms that, when secretion over $0-60 \mathrm{~min}$ is assessed, the concentration samples at $t_{7}=90 \mathrm{~min}$ and $t_{8}=120 \mathrm{~min}$ have marginal effects. In fact, the coefficients $\theta_{7}$ and $\theta_{8}$ are close to 0 for both LH and FSH. Moreover, for FSH the solution to the equation depends almost entirely on the samples at times $t_{2}=0$ and $t_{6}=60$ (except for $\theta_{2}$ and $\theta_{6}$, all the other coefficients $\theta_{j}$ are small). This is not surprising as, because of the very long half-life of FSH, the serum FSH concentration is about equal to the integral of the FSH ISR. Hence, the integral of the ISR over $0-60 \mathrm{~min}$ (eq. [3]) is approximately equal to the difference between the serum concentrations at times 60 and $0 \mathrm{~min}$ - that is $\theta_{2} \cong-1, \theta_{6} \cong 1, \theta_{j}=0, j \neq 2, j \neq 6$ (see equation [4]). In fact, the two-sample formula for FSH based on two samples at time 0 and $60 \mathrm{~min}\left(\theta_{1}=-0.9281\right.$ and $\theta_{2}=1.1402$ ) gives fully satisfactory results (data not shown).

\section{Discussion}

In the past decade, the use of deconvolution analysis in the investigation of hormone data has been the object of several studies. Different approaches have been implemented ranging from discrete deconvolution (1, $2,4,14,15)$, to parametric $(3,16)$ and non-parametric deconvolution, $(5-8,17)$. As discussed earlier (8), the non-parametric approach is particularly well suited to the analysis of RTS (response-to-stimuli) hormone data.

In the absence of an established mathematical model of the ISR profile allowing for the type and size of the stimulus response, it may be useful to apply a method that does not introduce undue assumptions about the secretory pattern. The only assumption underlying non-parametric deconvolution concerns the 

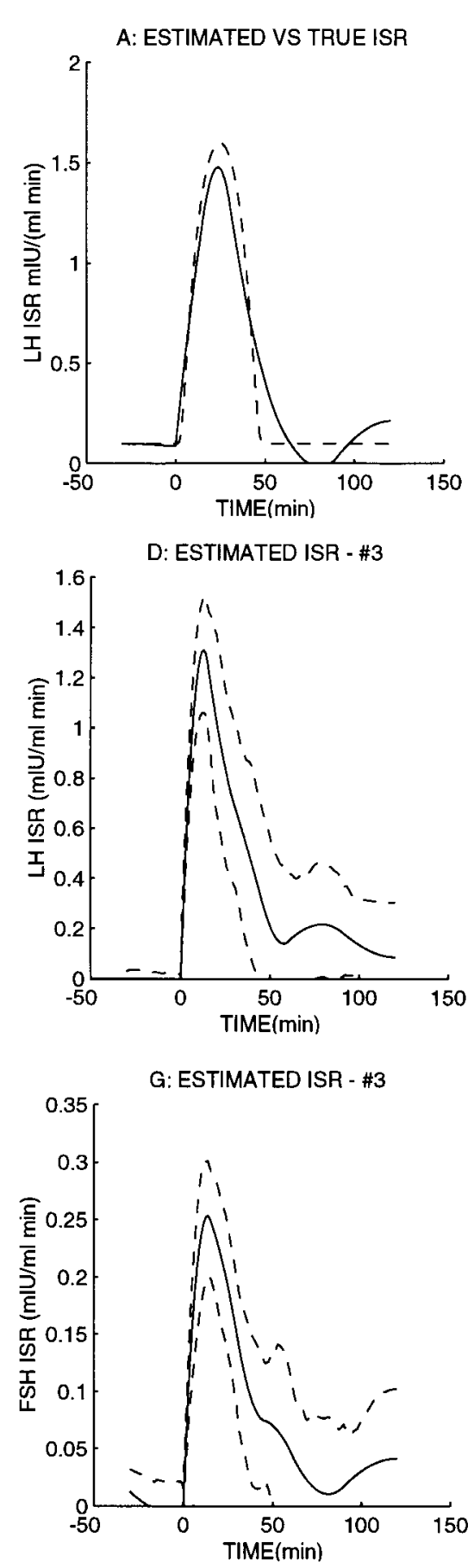

B: LH CONFIDENCE LIMITS
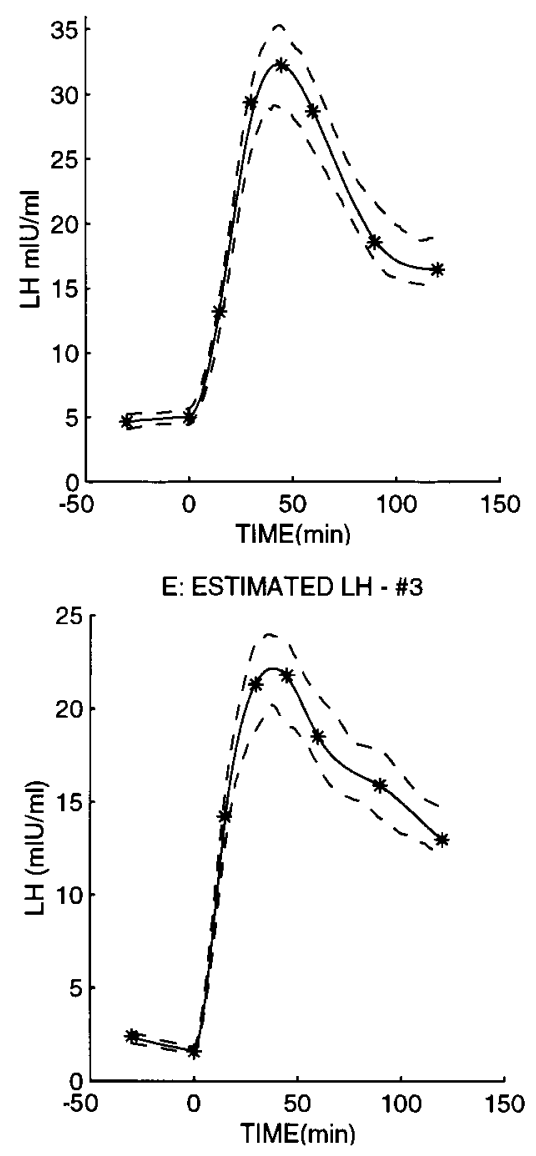

H: ESTIMATED FSH - \#3

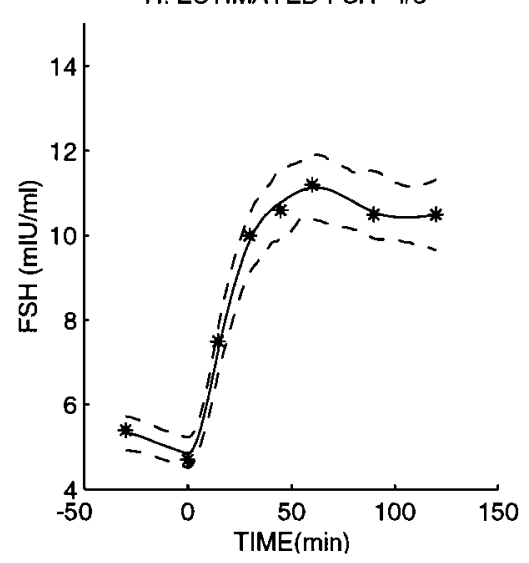

C: PERTURBED ISR ESTIMATES

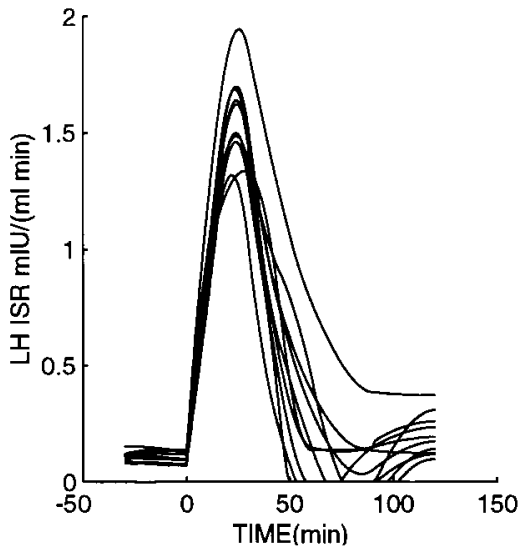

F: PERTURBED ISR ESTIMATES - \#3
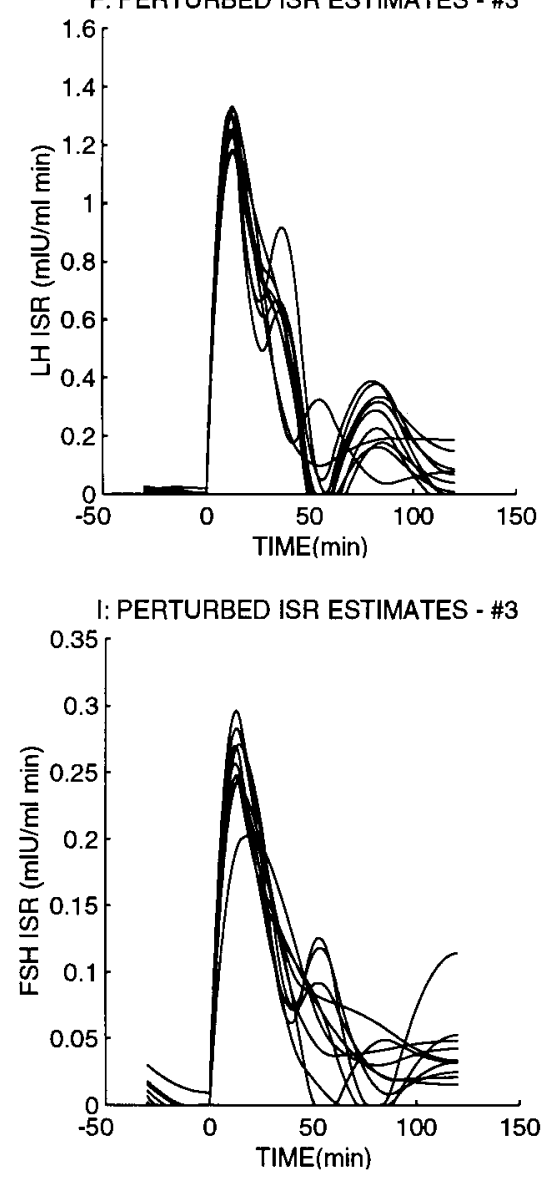

Figure 2 Deconvolution of simulated and clinical data. (A-C) Simulated benchmark problem for LH. A: ISR estimated by deconvolution ISR (dashed line), and true ISR (continuous line); B: reconvoluted LH concentration with $95 \%$ confidence limits allowing for both measurement error and kinetic model uncertainty; C: 10 estimated ISR profiles extracted from a sample space representative of the uncertainty affecting the estimates. (D-F) LH response to a single GnRH bolus. D and E: estimated ISR and reconvoluted LH concentration with $95 \%$ confidence limits allowing for both measurement error and kinetic model uncertainty; F: 10 estimated ISR profiles extracted from a sample space representative of the uncertainty affecting the estimates. (G-I) FSH response to a single $\mathrm{GnRH}$ bolus. $\mathrm{G}$ and $\mathrm{H}$ : estimated ISR and reconvolved LH concentration with $95 \%$ confidence limits allowing for both measurement error and kinetic model uncertainty; I: 10 estimated ISR profiles extracted from a sample space representative of the uncertainty affecting the estimates. 

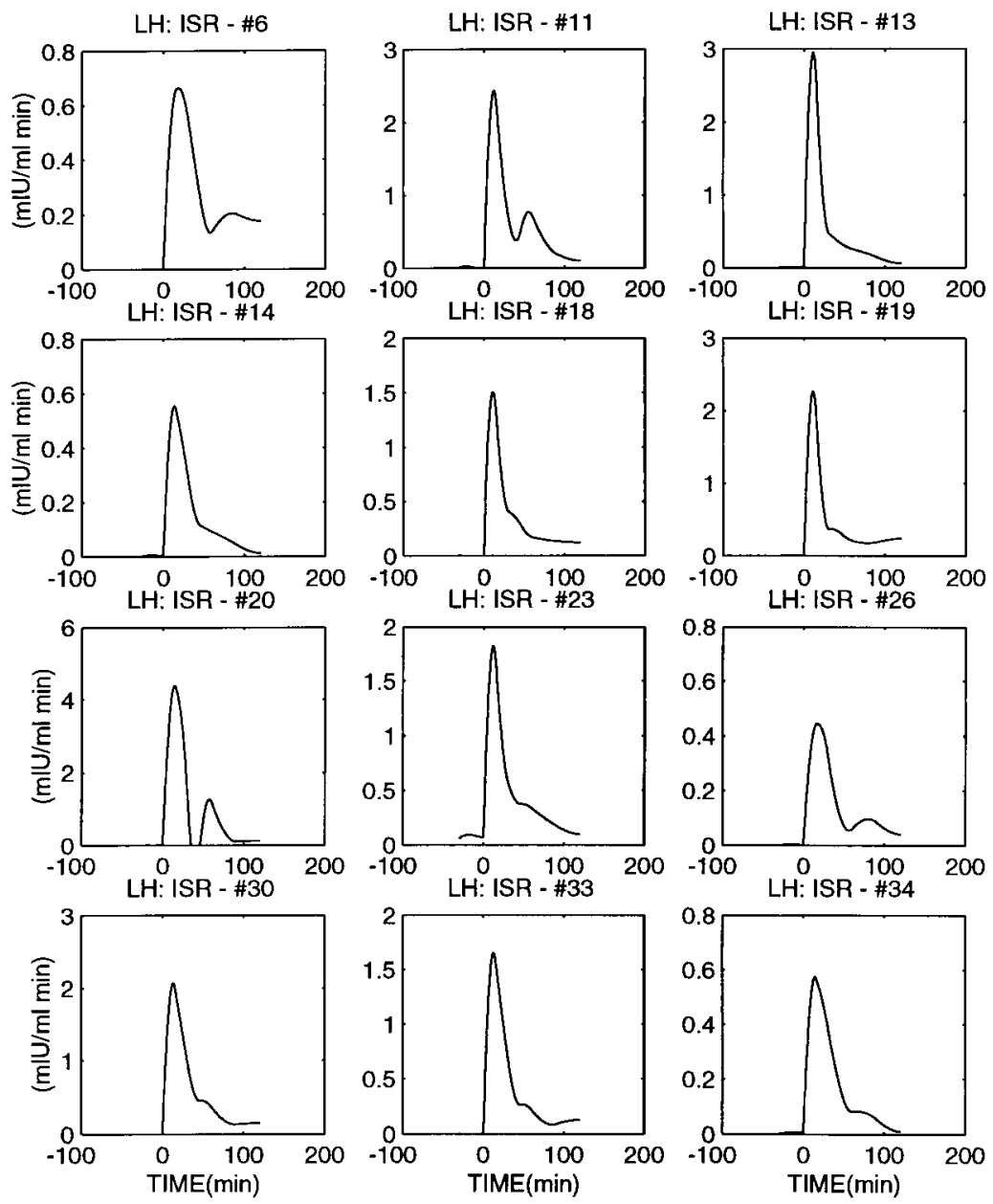

Figure $3 \mathrm{LH}$ secretory responses in 12 volunteers given a $\mathrm{GnRH}$ bolus at time 0 min: ISR profiles estimated by deconvolution analysis.

'smoothness' of the ISR. In this respect, a regularization parameter that controls the balance between smoothness and fit to data can be adjusted by means of an objective statistical criterion, such as the maximum likelihood method.

The use of non-parametric deconvolution in the analysis of RTS data is a recent innovation. GH responses to GH-releasing stimuli have been studied in this way $(6,8)$, and we have now applied the same methodology to the analysis of LH and FSH responses to GnRH stimuli. Although previous studies validated the non-parametric deconvolution algorithm through computer-simulated experiments, they did not address the issue of in vivo validation. In the present study, the deconvolution algorithm was validated by using it for reconstructing the doses injected in healthy volunteers whose LH secretion had been suitably downregulated. The very good agreement, over a 10-fold range, between the true doses and the reconstructed values provides a convincing in vivo validation of non-parametric deconvolution that was not available before.

Concerning the RTS data, a first notable result is that, although GH, LH and FSH exhibited different serum concentration profiles, their ISR profile was qualitatively the same: a primary secretory pulse started just after the stimulus and lasted about $60 \mathrm{~min}$. This means that these hormones are secreted in much the same way by the pituitary gland, and that the apparent differences in serum concentration profiles are explained only by the different half-lives, GH having the shortest and FSH the longest among the three hormones (about $10 \mathrm{~min}$ for $\mathrm{GH}, 30 \mathrm{~min}$ for $\mathrm{LH}$ and $200 \mathrm{~min}$ for $\mathrm{FSH}$ ). This was also an inference from an earlier waveform-independent methodology, when applied to spontaneous 24 -h pulse profiles of GH, LH, FSH, prolactin, ACTH, $\beta$-endorphin and TSH (18).

The results of the present study are of immediate importance in assessing the responsiveness of the pituitary gland to $\mathrm{GnRH}$ stimuli. In general, two approaches are possible. The first is to consider the peak value of the serum concentration profile. However, a peak-based assessment may be unsatisfactory for the following reasons: (i) it is based on a single sample; (ii) as a rule, the true peak does not coincide with the arbitrary moment(s) of sampling; (iii) it does not directly evaluate the amount of hormone secreted by the gland. 
Figure 4 FSH secretory responses in 12 volunteers given a $\mathrm{GnRH}$ bolus at time $0 \mathrm{~min}$ : ISR profiles estimated by deconvolution analysis.
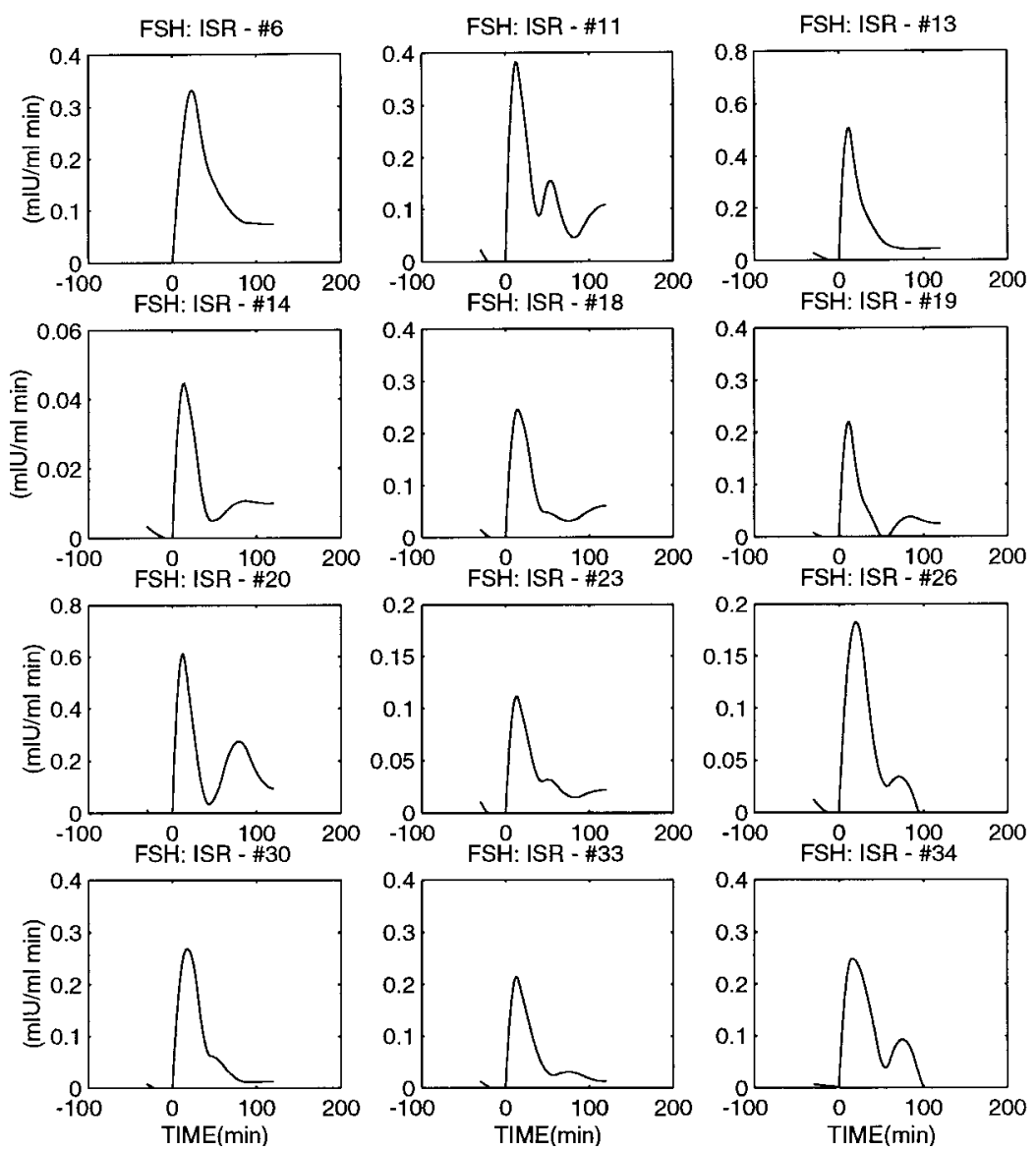

Alternatively, one can resort to a measure of integral type, such as the incremental AUC, which takes into account the entire serum concentration profile (see, for example, (19)). However, this second approach is viable only for hormones having relatively short half-lives, such as GH and ACTH. In fact, the incremental AUC is roughly proportional to the glandular secretory response, measured in terms of released hormone, only if: (i) the sampling schedule covers the complete descending limb of the serum concentration response; (ii) no spontaneous secretion pulse occurs concomitantly. When hormones with relatively long half-lives, such as LH and FSH, are being considered, these conditions cannot be satisfied, because $120 \mathrm{~min}$ is an insufficient period in which to capture the complete descending limb (e.g. slow components of $\mathrm{LH}$ and FSH removal are 90 and $500 \mathrm{~min}$, approximately). Conversely, the use of a longer sampling schedule not only would be more expensive, but would also increase the probability of occurrence of spontaneous pulses.

The present study has demonstrated that deconvolution analysis with literature-based hormone half-life values makes it possible to assess the entire amount of released $\mathrm{LH}$ (or $\mathrm{FSH}$ ) without the need to observe the complete descending limb of the concentration peak.
Deconvolution can provide this information because it reconstructs the time profile of the ISR using the serum concentration samples and a model of the hormone kinetics (summarized by the clearance parameters, e.g. of a biexponential form) (20). As the results show that, in normal individuals, most of the gonadotrophin secretory response of the pituitary gland occurs within 60 min after the GnRH stimulus, it is sensible to use the amount of hormone secreted in such a time interval as an inferential measure of glandular responsiveness to $\mathrm{GnRH}$.

Although the proposed deconvolution algorithm is computationally efficient, an ad hoc computer program is needed to perform the involved calculations. Therefore, a simple and direct integration formula for the responsiveness index has been devised. The equation is simply a linear combination of the serum concentration values assigned suitable weightings, which can be tabulated once for all, and which depend on the clearance parameters of the specific hormone and the sampling schedule. A comparison with the estimates obtained by deconvolution shows that the direct integration formula is very reliable.

Inspection of the weightings of the direct integration formula reveals that the contribution of the samples at 90 and $120 \mathrm{~min}$ is negligible in normal individuals. 

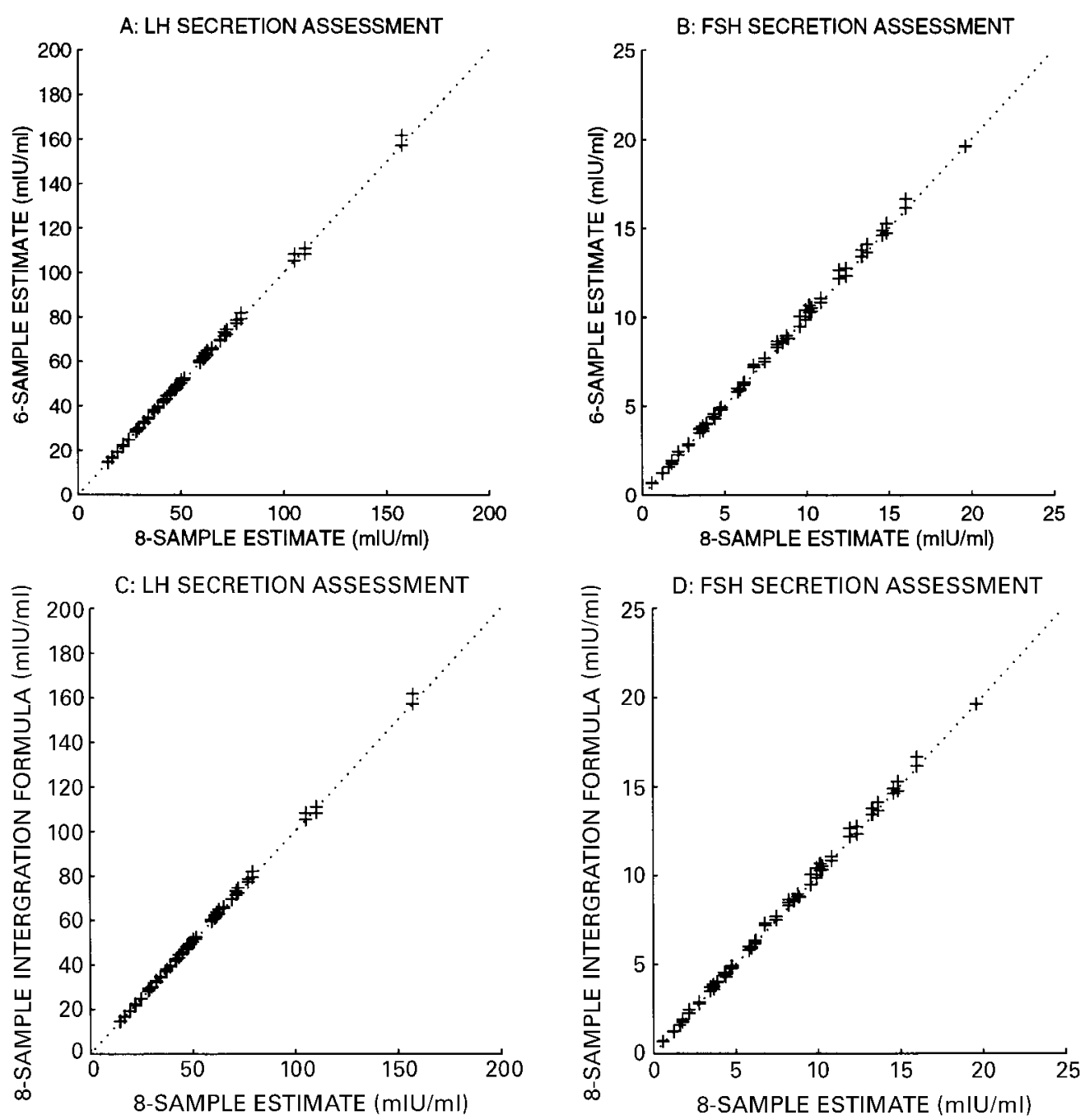

Figure $5 \mathrm{LH}$ and FSH response to a single GnRH bolus: assessment of the cumulated ISR over 0-60 min. (A, B) Comparison between the cumulated ISR over 0-60 min estimated using either eight or six samples (LH and FSH). When six samples were used, the data at times 90 and $120 \mathrm{~min}$ were discarded. (C, D) Comparison between deconvolution using eight samples and the eight-sample quadratic equation (LH and FSH).

Therefore, as far as the estimation of the cumulative secretory response between 0 and 60 min is concerned, the last two samples can be omitted, and the duration of the experiment reduced. This confirms what had already been observed in the context of GH responses to stimuli (8). By use of the direct integration formula, it is even possible to obtain a fairly accurate evaluation of the amount of secreted FSH within 60 min of the stimulus using only two serum FSH concentrations, at time 0 and $60 \mathrm{~min}$.

It could seem that the availability of the direct equation makes deconvolution superfluous. In reality, deconvolution analysis remains informative, as it evaluates not only the amount of secreted hormone, but also the mode of secretion - for example the shape and duration of the secretory spike. Hence, deconvolution analysis offers a powerful (non-invasive) method to inspect (pituitary) secretory mechanisms in various pathophysiological conditions. In this respect, the available results show that, in exogenously stimulated normal individuals, different hormones such a $\mathrm{GH}$, LH and FSH are secreted according to a common qualitative pattern.

In the present study, deconvolution analysis has been carried out using population values of the LH and FSH kinetic parameters. This introduces a further source of uncertainty, in addition to the measurement errors affecting the serum concentration data. Both sources have been taken into account in the computation of the confidence intervals by means of a Monte Carlo procedure. This procedure generates randomly perturbed problems, the solutions to which are used statistically to assess the desired error bars. Although the availability of patient-specific kinetic parameters 
obtained from a separate experiment would increase the reliability of the estimated ISR, such an exhaustive procedure cannot always be applied to the more routine analysis of clinical data. In any case, the validation study has demonstrated that these uncertainties do not impair too much the accuracy of the estimates.

In the present study, we sampled at a commonly used frequency of approximately every $15 \mathrm{~min}$, with two samples before the secretagogue injection. Our analysis does not preclude sampling at more frequent intervals, and also allows for irregularly spaced samples. Indeed, the latter strategy with more frequent samples just after the stimulus, may help identify with somewhat greater accuracy the absolute peak secretory rate achieved after the stimulus. The present studies are also qualified by their application to normal younger men, although we recognize no theoretical limitations to the implementation of the methodology for women of pre- and postmenopausal age, and children. However, in clinical circumstances of severely impaired hepatic or renal function, the investigator must obtain appropriate values for gonadotrophin half-lives, given the impaired clearance of these glycoprotein hormones in presence of functional liver or renal failure. We note that either LH or FSH secretory responses exhibit a broad quantitative spectrum, which is of an approximately 10-fold range in normal healthy younger populations. Lastly, when highly purified LH and FSH standards become available, it will eventually be useful to calculate the relative molar amount of LH and FSH secreted after varying GnRH stimuli (here, in different reference preparations, the LH secretory rate was approximately 10-fold that of $\mathrm{FSH}$ ).

\section{Appendix}

\section{Derivation of the integration formula for assessment of secretion}

In order to derive a simple and direct formula, two approximations are necessary. First, instead of using the modified score function that allows for discontinuity at time $0 \mathrm{~min}(8)$, the standard score function (eq. [2]) is introduced. Second, it is assumed that the regularization parameter provided by the maximum likelihood criterion is close to 0 (which, in the present problem, was confirmed by experience). When $\gamma$ tends to 0 , minimizing $J(s)$ given in equation [2] becomes equivalent to finding $s(t)$, which minimizes

$$
\int_{-\infty}^{t_{n}}\left(\frac{\mathrm{d} s(t)}{\mathrm{d} t}\right)^{2} \mathrm{~d} t
$$

subject to the constraints $y_{j}=c\left(t_{j}\right), j=1, n$. In order to obtain a numerical solution to such a problem, the convolution integral (eq. [1]) is made discrete. Letting T be the discrete period, it is assumed that the sampling instants $t_{j}$ are integer multiples of $T$. Note that $T$ can be chosen to be arbitrarily small, so that the error made by approximating the continuous function, $s(t)$, as a staircase function, can be made negligible. With this approximation, equation [1] becomes:

$$
y_{k}=\sum_{k=-M+1}^{N-M} g_{k-j} s(k T)+v_{k}, g_{i}=\int_{0}^{T} g(i T+T-\tau) \mathrm{d} \tau,
$$

or also, in matrix form, $y=G s+v$, where $y=\left[y_{1} y_{2} \ldots y_{n}\right]^{\prime}$, $v=\left[\begin{array}{lll}v_{1} & v_{2} \ldots v_{n}\end{array}\right]^{\prime}$ and $s=[s(-M T+1) \quad s(-M T+2) \ldots$ $s((N-M) T]^{\prime}$, and $G$ is a suitable $n \times N$ matrix. Note that $M$ must be sufficiently large to allow for border effects, the integer $N$ is such that $t_{n}=(N-M) T$, and the $N \times N$ matrix $V=S S^{\prime}$ is defined as:

$$
S=\left[\begin{array}{ccccc}
1 & 0 & 0 & \ldots & 0 \\
1 & 1 & 0 & \ldots & 0 \\
1 & 1 & 1 & \ldots & 0 \\
\ldots & \ldots & \ldots & \ldots & \ldots
\end{array}\right]
$$

The discretized solution to the constrained minimization of equation [A1] is $\hat{s}=V G^{\prime}\left(G V G^{\prime}\right)^{-1} y(6)$. Then, the estimate of the amount $H$ of secreted hormone is given by $H=\theta y$, where $\theta=R V G^{\prime}\left(G V G^{\prime}\right)^{-1}$, and $R=\left[\begin{array}{lll}r_{1} & r_{2} \ldots r_{n}\end{array}\right]$ is a row vector with

$$
r_{j}=\left\{\begin{array}{lc}
1 / T, & \text { if } 0 \leq j T<L \\
0, & \text { otherwise }
\end{array}\right.
$$

The weightings are given directly by the elements of the $n \times 1$ row vector, $\theta$.

\section{Acknowledgements}

This work was supported in part by: MURST project 'Algorithms and Architectures for the Identification and Control of Industrial Systems'; 'Progetti di Ricerca Corrente', Italian Institute for Auxology, Milano, Italy; NIH Grant No. RR11095-02; RCDA from NIHCD No. 1K04HD00634, NIH P30 Reproduction Research Center No. HD28934, and the NSF Center for Biological Timing.

Mr Pietro Prospiti is gratefully acknowledged for assistance in processing the data and preparing the manuscript.

\section{References}

1 Oerter KE, Guardabasso V \& Rodbard D. Detection and characterization of peaks and estimation of instantaneous secretory rate for episodic pulsatile hormone secretion. Computers and Biomedical Research 198619 170-191.

2 Cobelli C, Mari A, Del Prato S, De Kreutzenberg S, Nosadini R \& Jensen I. Reconstructing the rate of appearance of subcutaneous insulin by deconvolution. American Journal of Physiology 1987 252 E584-E590.

3 Veldhuis JD, Carlson ML \& Johnson ML. The pituitary gland secretes in bursts: appraising the nature of glandular secretory impulses by simultaneous multiple-parameter deconvolution of plasma hormone concentrations. Proceedings of the National Academy of Sciences of the USA 19874 7686-7690.

4 De Nicolao G \& Liberati D. Linear and nonlinear techniques for the deconvolution of hormone time-series. IEEE Transactions on Biomedical Engineering 199340 440-455. 
5 Johnson ML \& Veldhuis JD. Evolution of deconvolution analysis as a hormone pulse detection method. Methods in Neurosciences $1995281-24$

6 De Nicolao G, Liberati D \& Sartorio A. Deconvolution of infrequently sampled data for the estimation of growth hormone secretion. IEEE Transactions on Biomedical Engineering 199542 678-687.

7 De Nicolao G, Sparacino G \& Cobelli C. Nonparametric input estimation in physiological systems: problems, methods, and case studies. Automatica 199733 851-870.

8 Sartorio A, De Nicolao G, Pizzini G \& Liberati D. Non-parametric deconvolution provides an objective assessment of $\mathrm{GH}$ responsiveness to GH-releasing stimuli in normal subjects. Clinical Endocrinology 199746 387-395.

9 Pincus SM, Mulligan T, Iranmanesh A, Gheorghiu S, Godschalk M \& Veldhuis JD. Older males secrete luteinizing hormone and testosterone more irregularly, and jointly more asynchronously, than younger males. Proceedings of the National Academy of Sciences of the USA 199693 14100-14105.

10 Veldhuis JD, Fraioli F, Rogol AD \& Dufau ML. Metabolic clearance of biologically active luteinizing hormone in man. Journal of Clinical Investigation 198677 1122-1128.

11 Urban RJ, Padmanabhan V, Beitins I \& Veldhuis JD. Metabolic clearance of human follicle-stimulating hormone assessed by radioimmunoassay, immunoradiometric assay, and in vitro Sertoli cell bioassay. Journal of Clinical Endocrinology and Metabolism 199173 818-823.

12 Phillips DL. A technique for the numerical solution of certain integral equations of the first kind. Journal of the Association for Computing Machinery 19629 97-101.
13 Tikhonov AN \& Arsenin VY. Solutions of Ill-Posed Problems. Washington: Winston/Wiley 1977.

14 Van Cauter E \& Koninckx E. Pulsatility of pituitary hormones. Experimental Brain Research Supplement 198512 (Suppl) 41-60.

15 Veldhuis JD, Moorman J \& Johnson ML. Deconvolution analysis of neuroendocrine data: waveform-specific and waveform-independent methods and applications. Methods in Neurosciences 199420 279-325.

16 Veldhuis JD \& Johnson ML. Deconvolution analysis of hormone data. Methods in Enzymology 1992210 539-579.

17 Groth T, Rosberg S \& Albertsson-Wikland K. Estimation of growth hormone secretory patterns in children with use of a numerical deconvolution technique: experimental design with use of computer simulation. Hormone Research 199442 245-252.

18 Veldhuis JD, Iranmanesh A, Johnson ML \& Lizarralde G. Twentyfour hour rhythms in plasma concentrations of adenohypophyseal hormones are generated by distinct amplitude and/or frequency modulation of underlying pituitary secretory bursts. Journal of Clinical Endocrinology and Metabolism 199071 16161623.

19 Sartorio A, Spada A, Morabito F \& Faglia G. Different responsiveness to repeated GHRH administration in normal children and adults. Journal of Endocrinological Investigation 198811 727-729.

20 Veldhuis JD. How does one get at glandular secretion, when only hormone concentrations are measured? Clinical Endocrinology $199746397-400$.

Received 29 January 1999

Accepted 10 June 1999 